\title{
Correlations of ER, PR, Her-2 and CK5/6 expression with histological characteristics features and prognosis of breast ductal carcinoma in situ.
}

\author{
Aimin Ge*, Ting Hao, Weiyi Dong, Fangzheng Han, Huazhu Song \\ Department of Pathology, Heze Municipal Hospital, Heze, PR China
}

\begin{abstract}
This study aimed to investigate the correlations of Estrogen Receptor (ER), Progesterone Receptor (PR), human epidermal growth factor receptor 2 (Her-2) and cytokeratin 5/6 (CK5/6) protein expression with histological characteristics features and prognosis of breast Ductal Carcinoma in situ (DCIS). Fortyeight cases of breast DCIS specimen were enrolled in this study. The samples were histologically typed and graded. The immunohistochemical analysis was performed to determine the expression of ER, PR, Her-2 and CK5/6 protein. Results showed that, the positive rates of ER and PR protein expression in non-comedo type DCIS group were significantly higher than those in comedo type DCIS group, respectively $(\mathbf{P}<\mathbf{0 . 0 5})$, and the positive rate of $\mathrm{Her}-2$ protein expression in non-comedo type group was significantly lower than that in comedo type group $(\mathbf{P}<0.05)$. There was no significant difference of CK5/6 protein expression between two groups $(P>0.05)$. There was significant difference of positive rate of ER, PR, Her-2 protein expression among histological grades I-III $(\mathbf{P}<0.01)$, with no significant difference of $\mathrm{CK5} / 6$ protein expression among three groups $(\mathrm{P}>0.05)$. The expression of $E R$ protein was positively correlated with $P R$ protein expression $(r=0.764, P<0.001)$ and negatively correlated with Her-2 protein expression $(r=-0.643, P<0.001)$; the expression of $P R$ protein was negatively correlated with Her-2 protein expression $(r=-0.622, P<0.001)$. In 4 recurrent DCIS cases, Her-2 protein expression was positive in 3 cases. ER, $P R$ and $C K 5 / 6$ protein expressions were negative in all 4 cases. In conclusion, $E R$, PR, Her-2 protein expressions are significantly correlated with the histological types and histological grades of breast DCIS. The positive Her-2 protein expression is related to the prognosis of breast DCIS.
\end{abstract}

Keywords: Estrogen receptor, Progesterone receptor, Human epidermal growth factor receptor 2, Cytokeratin 5/6, Ductal carcinoma in situ.

Accepted on November 20, 2017

\section{Introduction}

Breast cancer is one of the most common malignant tumors in women, and its occurrence and development are closely related to the abnormal expression of sex hormone receptors and several oncogenes [1,2]. Ductal Carcinoma in situ (DCIS) is defined as malignant hyperplasia of mammary ductal epithelial cells which is confined to the intraductal basement membrane without invasion of stroma. The incidence of DCIS is low, accounting for about $5 \%$ of breast cancer [3]. With the development of large-scale breast cancer screening program and the extensive application of various early tumor diagnosis technologies, the detection rate of DCIS is becoming higher and higher [4]. The prognosis of DCIS is closely related to the histology of the tumor, but there is no universally accepted and reproducible method for the classification of DCIS. In the past, according to the structure of cancer tissue, DCIS is divided into comedo type, solid type, cribriform type, papillary type and micropapillary type [5]. However, as DCIS is of multi-type mixed structure, different observers often draw different conclusions about the same patient, and the diagnosis reproducibility is low. In addition, some researchers also simply divide DCIS into comedo type and non-comedo type [6]. At present, DCIS is often divided into low (grade I), medium (grade II) and high (grade III) differentiation type according to the atypia and arrangement polarity of tumor cells [7]. It is well known that, Estrogen Receptor (ER), Progesterone Receptor (PR), human epidermal growth factor receptor 2 (Her-2) and cytokeratin 5/6 (CK5/6) are the important indicators to guide the treatment and prognosis of many kinds of cancer, and the basis for the application of endocrine therapy and targeted drug therapy are originated from these indexes [8,9]. This study investigated the correlations of ER, PR, Her-2 and CK5/6 expression with clinicopathological features and prognosis of DCIS. The objective was to provide a basis for clinical diagnosis and treatment of DCIS. 


\section{Materials and Methods}

\section{Materials}

Forty-eight cases of breast DCIS specimen obtained by surgical resection in our hospital from January 2001 to January 2011 were enrolled in this study. All 48 breast DCIS patients were female. Their age was 30-69 y old, with the average age of $48.3 \pm 6.5 \mathrm{y}$. All patients had complete follow-up data. The specimens were regularly fixed with formaldehyde, embedded with paraffin, sectioned and stained with HE. DCIS was diagnosed by more than two pathologists according to WHO standard.

\section{Histological typing of breast DCIS}

According to the histological typing, breast DCIS was divided into comedo type and non-comedo type. The non-comedo type DCIS could be divided into three subtypes including papillary type, micropapillary type, cribriform type and solid type. For comedo type DCIS, the central region presented degeneration and necrosis, and became a mass of eosinophilic and nonstructural substance. In the peripheral area, the cancer cell polarity disappeared, and the cells were generally large, with round nucleus, deep dye, and common nuclear fission. For papillary and micropapillary type DCIS, the tubular expansion was obvious, and the lined abnormal cells presented papillary and micropapillary shape to the cavity, respectively, and there was no fibrous stroma in the papilla. For cribriform type DCIS, the intraductal cancer cells presented medium size, forming many circular cavities, like cribriforms. There was usually no necrosis, and even slight necrosis. The size and shape of the cells were consistent, and nuclear fission was common. For solid type DCIS, the duct was filled with solid cells, presenting different degrees of expansion.

\section{Histological grading of breast DCIS}

The histological grading of breast DCIS was performed according to the WHO Classification of Tumors of the Breast in 2012: grade I: grade 1-2 nucleus, no comedo-type necrosis; grade II: grades 1 and 2 nucleus, with comedo-type necrosis; grade III: grade 3 nucleus. Grade 1 nucleus: the diameter of nucleus was 1-1.5 times of red blood cells, even chromatin, no nucleolus; grade 2 nucleus: the diameter of nucleus was 1-2 times of red blood cells, coarse chromatin, uncommon nucleolus; grade 3 nucleus: the diameter of nucleus was $>2$ times of red blood cells, with vesicular nuclei and two or more nucleoli.

\section{Immunohistochemical analysis}

DCIS tissue paraffin sections were prepared and normally dewaxed, followed by enzyme closure with $3 \%$ hydrogen peroxide and antigen retrieval with citrate buffer. After closing non-specific sites using non-immune goat serum, primary antibody with 1:50 dilution using PBS was added, followed by incubation at $4{ }^{\circ} \mathrm{C}$ overnight. After adding polymer enhancer and PBS washing, $50 \mu \mathrm{l}$ of horseradish peroxidase-labeled secondary antibody polymer was drop wisely added to each section, followed by incubation at $37^{\circ} \mathrm{C}$ for $30 \mathrm{~min}$ and $\mathrm{PBS}$ washing for 3 times. After DBA coloration, counterstain and mounting, the sections were observed in Q550CW image acquisition and analysis system (Leica Science Lab, Berlin, Germany).

\section{Judgment of staining results}

The staining results were judged by more than two pathologists. The positive ER and PR staining was localized at nuclei. Using $1 \%$ as the positive boundary value, $\geq 1 \%$ nuclei staining was judged as positive. In the case of positive control, $<1 \%$ nuclei staining was judged as negative. The positive Her-2 staining was localized at cell membrane. According to ASO/CAP scoring system [1], the staining scores were divided into $0,(+),(++)$ and $(+++)$ according to staining strength. 0 and $(+)$ presented negative; $(+++)$ presented positive. $(++)$ was not sure, and needed to be detected using fluorescence in situ hybridization (DISH). The Her-2 gene amplification presented the positive for HER-2 staining. The positive CK5/6 staining was located on the cytoplasm. $\geq 5 \%$ cell staining was judged as positive, and $<5 \%$ cell staining presented the negative reaction.

\section{Statistical analysis}

All statistical analysis was carried out using SPSS 20.0 software (SPSS Inc., Chicago, IL, USA). The enumeration data were presented as number and rate, and the comparison between two groups was performed using $\chi^{2}$ test. The correlation between two indexes was analysed by Spearman rank correlation analysis. $\mathrm{P}<0.05$ was considered as statistically significant.

\section{Results}

\section{Pathological characteristics of breast DCIS}

The histological typing showed that, among the 48 cases of breast DCIS, the cribriform type was the most common form, which accounted for $35.4 \%$ (17/48), followed by the comedo type $(31.3 \%, 15 / 48)$, papillary type $(14.6 \%, 7 / 48)$, micropapillary type $(10.4 \%, 5 / 48)$, and solid type $(8.3 \%, 4 / 48)$. The histological grading indicated that, among the 48 cases of breast DCIS, histological grades I-III accounted for $29.17 \%$ (14/48), 37.50\% (18/48), and 33.33 (16/48), respectively.

\section{Expressions of ER, PR, Her-2 and CK5/6 protein in breast DCIS with different histological types}

In 33 cases of breast DCIS with non-comedo type, the positive rates of ER, PR, Her-2 and CK5/6 protein expression were $78.8 \%, 66.7 \%, 33.3 \%$ and $12.1 \%$, respectively, and those in 15 cases of breast DCIS with comedo type were $46.7 \%, 33.3 \%$, $73.3 \%$ and $13.3 \%$, respectively. The positive rates of ER and PR protein expression in non-comedo type group were significantly higher than those in comedo type group, respectively $(\mathrm{P}<0.05)$, and the positive rate of Her- 2 protein expression in non-comedo type group was significantly lower 
Correlations of ER, PR, Her-2 and CK5/6 expression with histological characteristics features and prognosis of breast ductal carcinoma in situ

than that in comedo type group $(\mathrm{P}<0.05)$. There was no significant difference of positive rate of $\mathrm{CK} 5 / 6$ protein expression between two groups $(\mathrm{P}>0.05)$ (Table 1$)$.

Table 1. Expressions of ER, PR, Her-2 and CK5/6 protein in breast DCIS with different histological types.

\begin{tabular}{|c|c|c|c|c|c|c|}
\hline \multicolumn{3}{|c|}{ Histological type } & \multirow{2}{*}{$\begin{array}{l}\text { Non-comedo } \\
(n=33)\end{array}$} & \multirow{2}{*}{$\begin{array}{l}\text { Comedo } \\
(n=15)\end{array}$} & \multirow[t]{2}{*}{$x^{2}$} & \multirow[t]{2}{*}{$\mathbf{P}$} \\
\hline ER & $-(n)$ & & & & & \\
\hline & $+(n)$ & & 26 & 7 & & \\
\hline & $\begin{array}{l}\text { Positive } \\
(\%)\end{array}$ & rate & 78.8 & 46.7 & 4.953 & 0.026 \\
\hline \multirow[t]{3}{*}{ PR } & $-(n)$ & & 11 & 10 & & \\
\hline & $+(n)$ & & 22 & 5 & & \\
\hline & $\begin{array}{l}\text { Positive } \\
(\%)\end{array}$ & rate & 66.7 & 33.3 & 4.656 & 0.031 \\
\hline \multirow[t]{3}{*}{ Her-2 } & $-(n)$ & & 22 & 4 & & \\
\hline & $+(n)$ & & 11 & 11 & & \\
\hline & $\begin{array}{l}\text { Positive } \\
(\%)\end{array}$ & rate & 33.3 & 73.3 & 6.646 & 0.01 \\
\hline CK5/6 & $-(n)$ & & 32 & 14 & & \\
\hline
\end{tabular}

\begin{tabular}{lllll}
\hline$+(n)$ & 1 & 1 & & \\
\hline $\begin{array}{l}\text { Positive } \\
(\%)\end{array}$ & rate 3 & 6.7 & 0.342 & 0.559 \\
\hline
\end{tabular}

DCIS: Ductal Carcinoma In Situ; ER: Estrogen Receptor; PR: Progesterone Receptor; Her-2: Human Epidermal Growth Factor Receptor 2; CK5/6: Cytokeratin $5 / 6$.

\section{Expressions of ER, PR, Her-2 and CK5/6 protein in non-comedo type breast DCIS with different subtypes}

In 7 cases of breast DCIS with papillary type, the positive rates of ER, PR, Her-2 and CK5/6 protein expression were $85.7 \%$, $71.4 \%, 14.3 \%$ and $0.0 \%$, respectively. In 5 cases of breast DCIS with micropapillary type, the positive rates of ER, PR, Her-2 and CK5/6 protein expression were $80.0 \%, 80.0 \%$, $40.0 \%$ and $20.00 \%$, respectively. In 17 cases of breast DCIS with cribriform type, the positive rates of ER, PR, Her-2 and CK5/6 protein expression were $84.4 \%, 70.6 \%, 35.3 \%$ and $5.9 \%$, respectively. In 4 cases of breast DCIS with solid type, the positive rates of $\mathrm{ER}, \mathrm{PR}, \mathrm{Her}-2$ and $\mathrm{CK} 5 / 6$ protein expression were $50.0 \%, 25.0 \%, 50.0 \%$ and $0.0 \%$, respectively. There was no significant difference of positive rate of each protein expression among three groups $(\mathrm{P}>0.05)$ (Table 2$)$.

Table 2. Expressions of ER, PR, Her-2 and CK5/6 protein in breast DCIS with non-comedo type.

\begin{tabular}{|c|c|c|c|c|c|c|c|}
\hline Type & & Papillary $(n=7)$ & Micropapillary $(n=5)$ & Cribriform $(n=17)$ & Solid $(n=4)$ & $x^{2}$ & $\mathbf{P}$ \\
\hline \multirow[t]{3}{*}{ ER } & $-(n)$ & 1 & 1 & 3 & 2 & & \\
\hline & $+(n)$ & 6 & 4 & 14 & 2 & & \\
\hline & Positive rate (\%) & 85.7 & 80 & 82.4 & 50 & 2.318 & 0.509 \\
\hline \multirow[t]{3}{*}{ PR } & $-(n)$ & 2 & 1 & 5 & 3 & & \\
\hline & $+(n)$ & 5 & 4 & 12 & 1 & & \\
\hline & Positive rate (\%) & 71.4 & 80 & 70.6 & 25 & 3.714 & 0.294 \\
\hline \multirow[t]{3}{*}{ Her-2 } & $-(n)$ & 6 & 3 & 11 & 2 & & \\
\hline & $+(n)$ & 1 & 2 & 6 & 2 & & \\
\hline & Positive rate (\%) & 14.3 & 40 & 35.3 & 50 & 1.772 & 0.621 \\
\hline \multirow[t]{3}{*}{$\mathrm{CK} 5 / 6$} & $-(n)$ & 7 & 5 & 16 & 4 & & \\
\hline & $+(n)$ & 0 & 0 & 1 & 0 & & \\
\hline & Positive rate (\%) & 0 & 0 & 5.9 & 0 & 0.971 & 0.808 \\
\hline
\end{tabular}

DCIS: Ductal Carcinoma In Situ; ER: Estrogen Receptor; PR: Progesterone Receptor; Her-2: Human Epidermal Growth Factor Receptor 2; CK5/6: Cytokeratin 5/6.

\section{Expressions of ER, PR, Her-2 and CK5/6 protein in breast DCIS with different histological grades}

Table 3 showed that, in 14 cases of breast DCIS with histological grade I, the positive rates of ER, PR, Her-2 and CK5/6 protein expression were $92.9 \%, 78.6 \%, 28.6 \%$ and $7.1 \%$, respectively. In 18 cases of breast DCIS with histological grade II, the positive rates of ER, PR, Her-2 and
CK5/6 protein expression were $83.3 \%, 72.2 \%, 33.3 \%$ and $5.6 \%$, respectively. In 16 cases of breast DCIS with histological grade III, the positive rates of ER, PR, Her-2 and CK5/6 protein expression were $31.3 \%, 18.8 \%, 75.0 \%$ and $0.0 \%$, respectively. There was significant difference of positive rate of ER, PR, Her-2 protein expression among three groups 
$(\mathrm{P}<0.01)$, with no significant difference of positive rate of

CK5/6 protein expression among three groups $(\mathrm{P}>0.05)$.

Table 3. Expressions of ER, PR, Her-2 and CK5/6 protein in breast DCIS with different histological grades.

\begin{tabular}{|c|c|c|c|c|c|c|}
\hline Histological grade & & Grade I ( $n=14)$ & Grade II $(n=18)$ & Grade III (n=16) & $x^{2}$ & $\mathbf{P}$ \\
\hline \multirow[t]{3}{*}{ ER } & $-(n)$ & 1 & 3 & 11 & & \\
\hline & $+(n)$ & 13 & 15 & 5 & & \\
\hline & Positive rate (\%) & 92.9 & 83.3 & 31.3 & 16.042 & $<0.001$ \\
\hline \multirow[t]{3}{*}{ PR } & $-(n)$ & 3 & 5 & 13 & & \\
\hline & $+(n)$ & 11 & 13 & 3 & & \\
\hline & Positive rate (\%) & 78.6 & 72.2 & 18.8 & 13.843 & $<0.001$ \\
\hline \multirow[t]{3}{*}{ Her-2 } & $-(n)$ & 10 & 12 & 4 & & \\
\hline & $+(n)$ & 4 & 6 & 12 & & \\
\hline & Positive rate (\%) & 28.6 & 33.3 & 75 & 8.296 & 0.016 \\
\hline \multirow[t]{3}{*}{ CK5/6 } & $-(n)$ & 13 & 17 & 16 & & \\
\hline & $+(n)$ & 1 & 1 & 0 & & \\
\hline & Positive rate (\%) & 7.1 & 5.6 & 0 & 0.093 & 0.579 \\
\hline
\end{tabular}

DCIS: Ductal Carcinoma In Situ; ER: Estrogen Receptor; PR: Progesterone Receptor; Her-2: Human Epidermal Growth Factor Receptor 2; CK5/6: Cytokeratin 5/6.

\section{Correlations of ER, PR, Her-2 and CK5/6 protein expression in breast DCIS}

Spearman rank correlation analysis showed that, in breast DCIS $\mathrm{s}$, the expression of ER protein was positively correlated with PR protein expression $(\mathrm{r}=0.764, \mathrm{P}<0.001)$ and negatively correlated with Her-2 protein expression $(\mathrm{r}=-0.643, \mathrm{P}<0.001)$; the expression of PR protein was negatively correlated with Her-2 protein expression $(\mathrm{r}=-0.622, \mathrm{P}<0.001)$. There was no significant correlation between each other two indexes (Table 4).

Table 4. Correlations of ER, PR, Her-2 and CK5/6 protein expression in breast DCIS.

\begin{tabular}{llllll}
\hline Index & & ER & PR & Her-2 & CK5/6 \\
\hline ER & $r$ & 1 & 0.764 & -0.643 & 0.141 \\
\cline { 2 - 6 } & $\mathrm{P}$ & - & 0 & 0 & 0.341 \\
\hline PR & $\mathrm{r}$ & 0.764 & 1 & -0.622 & 0.184 \\
\cline { 2 - 6 } & $\mathrm{P}$ & 0 & - & 0 & 0.211 \\
\hline Her-2 & $\mathrm{r}$ & -0.643 & -0.622 & 1 & 0.017 \\
\cline { 2 - 6 } & $\mathrm{P}$ & 0 & 0 & - & 0.906 \\
\hline
\end{tabular}

DCIS: Ductal Carcinoma In Situ; ER: Estrogen Receptor; PR: Progesterone Receptor; Her-2: Human Epidermal Growth Factor Receptor 2; CK5/6: Cytokeratin 5/6.

\section{Relations of ER, PR, Her-2 and CK5/6 protein expression with prognosis of breast DCIS}

In 48 breast DCIS patients, 20 cases underwent simple radical mastectomy, and 14 cases were treated with modified radical mastectomy. No recurrence occurred after operation in these 24 cases. The five-year survival rate of them was $100 \%$. Other 14 cases received the excision of local lesion, among them 4 cases suffered from the recrudescence within 6-18 months after surgery, including 1 case developing invasive ductal carcinoma (IDC). In these 4 recurrent cases, the histological grades I-III were in 1, 2 and 1 cases, respectively. In these 4 recurrent cases, Her-2 protein expression was positive in 3 cases. ER, PR and CK5/6 protein expressions were negative in all 4 cases.

\section{Discussion}

DCIS is a neoplastic proliferation of ductal epithelium, but not beyond the basement membrane [10]. Although the prognosis of most DCIS patients is relatively good, DCIS does not represent a single entity [11]. Until now the ideal treatment for DCIS has not been found. Studies have shown that, some DCIS patients receiving conservative surgery will suffer from the recurrence, and about half of the patients with recurrence take surgical treatment the same with the IDC [12]. Evenly after neoadjuvant radiotherapy, there is nearly $10 \%$ recurrence rate of DCIS, and about half of the cases develop into the IDC after 
recurrence [13]. It is found that, the relative risk rate of DCIS transformation into IDC is $8 \%-10 \%$ [14]. Although there are extensive studies on DCIS, little is known about the biological behavior of this precancerous lesion. Therefore, studying the pathology and biological behavior heterogeneity of DCIS can not only help to improve the accuracy of pathological diagnosis, but also provide the prognostic indicators for DCIS recurrence or potential malignancy, conducive to the treatment. This study has investigated the correlations of ER, PR, Her-2 and CK5/6 expression with histological characteristic features and prognosis of DCIS. It can provide a basis for clinical diagnosis and treatment of DCIS.

ER and PR are sex hormone receptors in normal mammary epithelial cells. When cells become cancerous, ER and PR decrease or disappear [15]. Previous study shows that, ER and PR are expressed in the most part of DCIS cases. The high expressions of ER and PR are related to the low-grade DCIS [16]. Results of this study showed that, the positive rates of ER and PR protein expression in non-comedo type DCIS group were significantly higher than those in comedo type DCIS group, respectively $(\mathrm{P}<0.05)$. There was significant difference of positive rate of ER and $\mathrm{PR}$ protein expression among histological grades I-III $(\mathrm{P}<0.01)$. This indicates that, the expressions of ER and PR protein are related with histological types and histological grades of DCIS.

Her-2 gene is located in the q21 region of chromosome 17. It is the proto-oncogene which encodes Her-2/neu protein, and is usually inactive. The over-expression or amplification of the Her-2 gene can cause the recurrence of breast cancer, leading to a poor prognosis [17]. The expression of Her-2 in DCIS is higher than that in IDC [18]. CK5/6 is the $\alpha$-type fiber peptide which is specifically expressed in epithelial cells and cancer cells. CK5/6-positive tumors have with features of basal-like breast cancer. It reflects the tumor origin, but does not change with the tumor development [19]. Kristiina et al. [20] have detected 72 cases of primary breast cancer and found that, the positive rate of $\mathrm{CK} 5 / 6$ expression is $31 \%$, but it in secondary breast cancer is only $11 \%$. Results of this study showed that, the positive rate of Her-2 protein expression in non-comedo type group was significantly lower than that in comedo type group $(\mathrm{P}<0.05)$, with no significant difference of positive rate of $\mathrm{CK} 5 / 6$ protein expression between two groups $(\mathrm{P}>0.05)$. In addition, there was significant difference of Her-2 protein expression among histological grades of DCIS $(\mathrm{P}<0.01)$, with no significant difference of positive rate of $\mathrm{CK} 5 / 6$ protein expression $(\mathrm{P}>0.05)$. This indicates that, the expression of Her-2 protein is related with histological types and histological grades of DCIS. CK5/6 protein expression has no significant relation with histological types and histological grades of DCIS. This may be due to the relatively small sample size in this study.

This study also analysed the correlations of ER, PR, Her-2 and CK5/6 protein expression in breast DCIS and their relations with prognosis of breast DCIS. Results showed that, in breast DCIS $\mathrm{s}$, the expression of ER protein was positively correlated with $\mathrm{PR}$ protein expression $(\mathrm{r}=0.764, \mathrm{P}<0.001)$ and negatively correlated with Her-2 protein expression $(\mathrm{r}=-0.643, \mathrm{P}<0.001)$; the expression of PR protein was negatively correlated with Her-2 protein expression $(\mathrm{r}=-0.622, \mathrm{P}<0.001)$. In addition, in 4 recurrent cases, Her-2 protein expression was positive in 3 cases. ER, PR and CK5/6 protein expressions were negative in all 4 cases. This indicates that, the Her- 2 protein expression is significantly negatively correlated with ER and PR expression, and the positive Her-2 expression is the most related to the poor prognosis of breast DCIS.

In conclusion, the ER, PR, Her-2 protein expressions are significantly correlated with the histological types and histological grades of breast DCIS. In addition, they are related to the prognosis of breast DCIS. The detection of these indexes has the guiding significance for understanding the malignancy of breast DCIS, guiding the individual treatment and evaluating the prognosis. The sample size of this study is relatively small. In our next studies, larger sample size will make the results more convincing. In addition, other affecting factors for breast DCIS should be considered.

\section{References}

1. Begg L, Kuller LH, Gutai JP, Caggiula AG, Wolmark N, Watson CG. Endogenous sex hormone levels and breast cancer risk. Genet Epidemiol 1987; 4: 233-247.

2. Osborne C, Wilson P, Tripathy D. Oncogenes and tumor suppressor genes in breast cancer: potential diagnostic and therapeutic applications. Oncologist 2004; 9: 361-377.

3. Curigliano G, Disalvatore D, Esposito A, Pruneri G, Lazzeroni M, Guerrieri-Gonzaga A, Luini A, Orecchia R, Goldhirsch A, Rotmensz N, Bonanni B, Viale G. Risk of subsequent in situ and invasive breast cancer in human epidermal growth factor receptor 2-positive ductal carcinoma in situ. Ann Oncol 2015; 26: 682-687.

4. Bourmaud A, Soler-Michel P, Oriol M, Regnier V, Tinquaut F, Nourissat A, Bremond A, Moumjid N, Chauvin F. Decision aid on breast cancer screening reduces attendance rate: results of a large-scale, randomized, controlled study by the DECIDEO group. Oncotarget 2016; 7: 12885-12892.

5. Bethwaite P, Smith N, Delahunt B, Kenwright D. Reproducibility of new classification schemes for the pathology of ductal carcinoma in situ of the breast. J Clin Pathol 1998; 51: 450-454.

6. van de Vijver MJ, Peterse JL, Mooi WJ, Wisman P, Lomans J, Dalesio O, Nusse R. Neu-protein overexpression in breast cancer. Association with comedo-type ductal carcinoma in situ and limited prognostic value in stage II breast cancer. N Engl J Med 1988; 319: 1239-1245.

7. Fujii H, Szumel R, Marsh C, Zhou W, Gabrielson E. Genetic progression, histological grade, and allelic loss in ductal carcinoma in situ of the breast. Cancer Res 1996; 56: 5260-5265.

8. Altundag K, Bulut N, Sari E, Harputluoglu H. The role of aromatase inhibitors in the management of ductal carcinoma in situ with an estrogen receptor-positive/ progesterone receptor-negative/Her-2/neu receptor-positive pattern. Am J Surg 2007; 194: 272-273. 
9. Sailer V, Luders C, Kuhn W, Pelzer V, Kristiansen G. Immunostaining of $\Delta \mathrm{Np} 63$ (using the p40 antibody) is equal to that of p63 and CK5/6 in high-grade ductal carcinoma in situ of the breast. Virchows Arch 2015; 467: 67-70.

10. Jedeszko C, Victor BC, Podgorski I, Sloane BF. Fibroblast hepatocyte growth factor promotes invasion of human mammary ductal carcinoma in situ. Cancer Res 2009; 69: 9148-9155.

11. Walling AD. Ductal carcinoma in situ of the breast: not a single entity. Am Fam Physician 1999; 59: 1026.

12. Deutsch M. Ductal carcinoma in situ recurrent on the chest wall after mastectomy. Clin Oncol (R Coll Radiol) 1999; 11: 61-62.

13. Holmberg L, Garmo H, Granstrand B, Ringberg A, Arnesson LG, Sandelin K, Karlsson P, Anderson H, Emdin S. Absolute risk reductions for local recurrence after postoperative radiotherapy after sector resection for ductal carcinoma in situ of the breast. J Clin Oncol 2008; 26: 1247-1252.

14. Hanley K, Wang J, Bourne P, Yang Q, Gao AC, Lyman G, Tang P. Lack of expression of androgen receptor may play a critical role in transformation from in situ to invasive basal subtype of high-grade ductal carcinoma of the breast. Hum Pathol 2008; 39: 386-392.

15. Edery M, Imagawa W, Larson L, Nandi S. Regulation of estrogen and progesterone receptor levels in mouse mammary epithelial cells grown in serum-free collagen gel cultures. Endocrinology 1985; 116: 105-112.

16. VandenBussche CJ, Cimino-Mathews A, Park BH, Emens LA, Tsangaris TN, Argani P. Reflex Estrogen Receptor
(ER) and Progesterone Receptor (PR) analysis of ductal carcinoma in situ (DCIS) in breast needle core biopsy specimens: an unnecessary exercise that costs the united states \$35 million/y. Am J Surg Pathol 2016; 40: 1090-1099.

17. Palmu S, Soderstrom KO, Quazi K, Isola J, Salminen E. Expression of C-KIT and HER-2 tyrosine kinase receptors in poor-prognosis breast cancer. Anticancer Res 2002; 22: 411-414.

18. Gunia SR, Patel MS, Mamounas EP. Pathologic complete response of HER-2 Neu-positive invasive ductal carcinoma and ductal carcinoma in situ following neoadjuvant chemotherapy plus trastuzumab: a case report and review of literature. Case Rep Surg 2012; 2012: 454273.

19. Bhargava R, Beriwal S, McManus K, Dabbs DJ. CK5 is more sensitive than CK5/6 in identifying the basal-like phenotype of breast carcinoma. Am J Clin Pathol 2008; 130: 724-730.

20. Joensuu K, Leidenius M, Kero M, Andersson LC, Horwitz KB, Heikkila P. ER, PR, HER2, Ki-67 and CK5 in early and late relapsing breast cancer-reduced CK5 expression in metastases. Breast Cancer (Auckl) 2013; 7: 23-34.

\section{*Correspondence to}

Aimin Ge

Department of Pathology

Heze Municipal Hospital

PR China 